\title{
Grain Growth Dominance on Complex Permittivity of $\mathrm{MgTiO}_{3}$
}

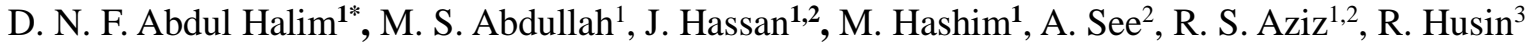 \\ Materials Synthesis and Characterization Laboratory (MSCL), Institute of Advanced Technology (ITMA), University Putra \\ Malaysia, 43400 UPM Serdang, Selangor, Malaysia \\ ${ }^{2}$ Department of Physics, Faculty of Science, University Putra Malaysia \\ 43400 UPM Serdang, Selangor, Malaysia \\ 3 Department of Physics, Faculty of Science, Universiti Technologi Malaysia \\ 81310 Skudai, Johor, Malaysia \\ ${ }^{*}$ Corresponding author's email: dayangnurfazliana [AT] gmail.com
}

\begin{abstract}
Uncovering the relationship between microstructural and dielectric properties is beneficial for designing new dielectric materials for technological purpose. Thus, this work attempts to understand the evolving relationship between complex permittivity and microstructural in magnesium titanate $\left(\mathrm{MgTiO}_{3}\right)$ at $40 \mathrm{~Hz}$ to $1 \mathrm{MHz}$. Magnesium oxide and titanium dioxide were mechanically crushed using a high energy ball mill for 12 hours via the mechanical alloying method. Pellets were formed followed by a sintering process from $500{ }^{\circ} \mathrm{C}$ up to $1400{ }^{\circ} \mathrm{C}$. The phase formations of the sintered samples showed a development in their crystalline structure and their phase was confirmed by X-ray diffraction patterns, yielding a completed phase of $\mathrm{MgTiO}_{3}$ formed at $800{ }^{\circ} \mathrm{C}$. Observation by scanning electron microscopy revealed an improvement in grain growth as the sintering temperatures are elevated. The density proportionally increased with the sintering temperature. From the complex permittivity studies, the dielectric constant, $\varepsilon_{r}$ ' showed a decreasing trend with increasing frequency and attained constant limitation value of $\varepsilon_{r}$ ' at higher frequency at room temperature. Below $10^{4} \mathrm{~Hz}$, the relaxation belonged to the interfacial polarization while beyond $10^{4} \mathrm{~Hz}$, it is dominant by dipolar polarization. The frequency dependence of loss tangent, tan $\delta$ decreased with value almost zero at higher frequency for all sintering temperatures. A significant increasing trend was observed which correlated the polarization-crystallinity behaviors at $1 \mathrm{MHz}$ from $500{ }^{\circ} \mathrm{C}$ up to $1400{ }^{\circ} \mathrm{C}$. The improvement in microstructure properties with respect to the sintering temperature was observed which give rise to the dielectric permittivity at infinite frequency, $\varepsilon_{\infty}$.' It revealed the dependency of dipolar polarization on the grain sizes and the crystallinity of the sample.
\end{abstract}

Keywords- Magnesium Titanate, Dielectric Response, Mechanical Alloying, Ceramic

\section{INTRODUCTION}

The current tendency in dielectric materials based on $\mathrm{TiO}_{2}$ is rising with a rapid development in electronic industry and satellite communications systems [1,2]. One of the promising materials is magnesium titanate, $\mathrm{MgTiO}_{3}$ which has good dielectric properties such as intermediate dielectric constant, $\varepsilon_{\mathrm{r}}{ }^{\prime}=15-20$, low dielectric loss and high $\mathrm{Q}$ values; $\mathrm{Q}=$ 20,000 at $10 \mathrm{Ghz}$ [2]. A number of researchers reported that the equilibrium phase of binary magnesium titanate showed existence of three stable phases $\mathrm{MgTiO}_{3}, \mathrm{Mg}_{2} \mathrm{TiO}_{4}$ and $\mathrm{MgTi}_{2} \mathrm{O}_{5}$ [3-6]. $\mathrm{MgTiO}_{3}$ has the ilmenite structure; $\mathrm{Mg}_{2} \mathrm{TiO}_{4}$ has the spinel structure and $\mathrm{MgTi}_{2} \mathrm{O}_{5}$ has the pseudobrookite structure. Thus, magnesium titanate has attracted much attention in microwave applications such as multilayer capacitor, band-pass filters, oscillators in radar detectors, cellular telephones and global positioning satellite devices [7, 8].

For the past thirty years, various methods have been used to synthesize these materials such as solid state reaction method [9-11], sol gel technique [12,13], hydrothermal mechano-chemical complexation routes [14-16] and stearic acid gel method [17]. Among the processes with the highest potential for tailoring advanced materials, mechanical alloying techniques are of special interest because they offer great flexibility in the choice of constituent materials to be combined, simplicity, relatively inexpensive to produce and etc. $[10,18]$. Mechanical treatment also can change the thermodynamic potentials, reducing sintering temperature, enhances the atomic mobility and therefore promotes different microstructures properties of material [19].

By manipulating the sample preparation technique and microstructure characteristics of magnesium titanate, great development of dielectric response study has been achieved. Ferreira and Baptista [12] correlated the structural and dielectric properties with the addition of dopants on magnesium titanate. The results showed that the dielectric loss is higher in doped sample rather than in pure sample due to the anharmonicity of the $\mathrm{MgTiO}_{3}$ lattice vibration and 
accomodation of dopants on $\mathrm{MgTiO}_{3}$. Miao et al. [13] concluded that uniform grain growth of a low porosity samples prepared via sol gel methods contribute to an excellent dielectric properties and good temperature stability.

Many reports have been made generally on the influence of composition [5], effect of dopants [20] and effect of milling time on $\mathrm{MgO}-\mathrm{TiO}_{2}$ systems [21]. However, fewer sufficient data available regarding the parallel evolution of microstructural and complex permittivity of the grain and grain boundaries of magnesium titanate prepared via mechanical alloying with starting material in nanometer size relating them at lower temperatures until they are evolving towards their final form and values. Therefore, we attempt to understand the relationship between microstructural and complex permittivity in magnesium titanate.

\section{EXPERIMENTAL}

The $\mathrm{MgTiO}_{3}$ ceramic samples were prepared via mechanical alloying technique. High purity of $\mathrm{MgO}$ (99.95\% purity, Alfa Aesar) and $\mathrm{TiO}_{2}$ (99.95\%, Alfa Aesar) as the starting materials were weighed according to the targeted proportion (molar ratio of magnesium oxide to titanium oxide is fixed at 1.025:1) and milled in SPEX SamplePrep 8000 Mixer for 12 hours in ambient temperature. The ball-to-powder mass-charge ratio (BPR) was set to 10:1. The ceramic powders were then compacted into a disk shaped samples under a pressure of $40 \mathrm{kPa}$ with $1 \%$ polyvinyl alcohol (PVA) as binder and zinc stearate as a lubricant. Each compacted pellets were sintered with different temperatures ranging from $500{ }^{\circ} \mathrm{C}$ to $1400{ }^{\circ} \mathrm{C}$ at $100^{\circ} \mathrm{C}$ increment.

The phase composition and crystal lattice parameters of sintered ceramics were examined by means of X-ray diffraction in $\mathrm{CuK} \alpha$ radiation using Philips Panalytical X-Ray Diffractometer equipped with diffraction software analysis. Diffraction patterns were collected in the range from 10 to $90^{\circ}$, in steps of $0.03^{\circ}$. Identification of the diffraction peaks of the XRD patterns was carried out by using the JCPDS database. The microstructures analysis of as-milled powder was employed by transmission electron microscope (LEO 912AB Energy Filter TEM), operating at $20 \mathrm{kV}$. The morphologies of the pellets were performed by means of Field Emission Scanning Electron Microscope (FESEM, FEI NOVA NanoSEM 230) operating at $10 \mathrm{kV}$ and the grain size was measured by the mean linear intercept method. The density of the sample was obtained using Electronic Densimeter MD300S which adopts Archimedes' principle. The dielectric properties were investigated using the Agilent 4294A Impedance/material analyzer in the region ranging from $40 \mathrm{~Hz}$ to $1 \mathrm{MHz}$ at room temperature.

\section{RESULTS AND DISCUSSION}

\subsection{Microstructural Analysis}

Fine powdered has proved to be very advantageous because of high strength, lowered the sintering temperature, high fracture toughness and improved the dielectric properties [22]. Therefore, a TEM micrograph of 12 hours as-milled powder is presented in Figure 1. It revealed small spherical shape particles with average grain size around $91 \mathrm{~nm}$. The particle size distribution of the raw materials is important since it affected the microstructure growth. Small particle size powder would offer a large surface energy to give a higher driving force for the crystalline growth [23]. As the thermal heat is applied, the free energy of the system decreased proportionally with the surface area. Thus, the grain growth and densification rate is increased with smaller particle size.

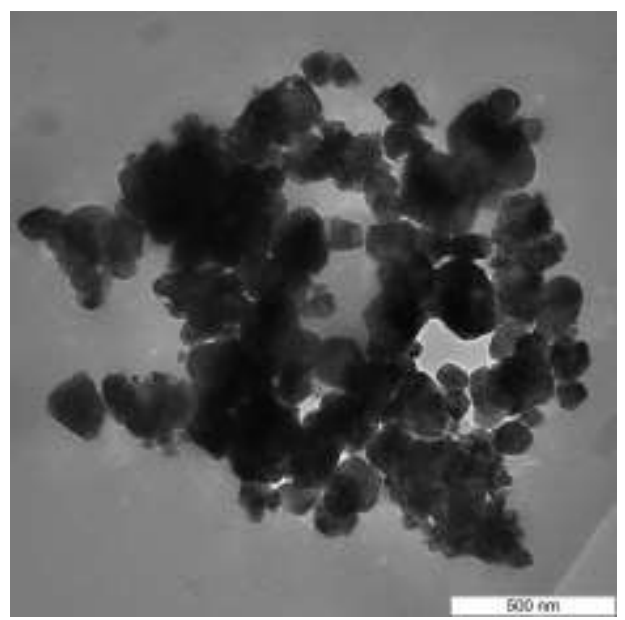

Figure 1: TEM micrograph of as-milled powder after 12 hours of milling 
The analysis of microstructure properties plays a vital role in the ongoing advancement of dielectric properties therefore in the performance of the products. Figure 2 shows the XRD patterns of $\mathrm{MgTiO}_{3}$ ceramic powders as a function of sintering temperature ranging from $500{ }^{\circ} \mathrm{C}$ to $1400{ }^{\circ} \mathrm{C}$.

The as-milled powder showed a presence of secondary phase, $\mathrm{Mg}_{2} \mathrm{TiO}_{5}$ and residual starting material phases, $\mathrm{TiO}_{2}$ and $\mathrm{MgO}$. The traced of $\mathrm{MgTiO}_{3}$ amorphous peak was not observed proved the alloying process cannot deformed the starting material $\mathrm{TiO}_{2}$ and $\mathrm{MgO}$ into desired $\mathrm{MgTiO}_{3}$ phase without heat treatment. The identification of all obtained patterns has been accomplished using ICDD database which indexed as $\mathrm{MgTiO}_{3}(00-006-0494), \mathrm{Mg}_{2} \mathrm{TiO}_{5}(01-079-0833)$, $\mathrm{TiO}_{2}(01-083-2243)$ and $\mathrm{MgO}(00-045-0946)$.

At early stage of sintering process, $\left(500{ }^{\circ} \mathrm{C}, 600{ }^{\circ} \mathrm{C}\right.$ and $\left.700{ }^{\circ} \mathrm{C}\right)$ the diffraction peaks corresponding to $\mathrm{MgTiO}_{3}$ slowly improved in the degree of crystallinity as the peaks became sharper with a higher intensity particularly at reflections of (104), (110), (113) and (116). At $600{ }^{\circ} \mathrm{C}$, new peaks of $\mathrm{MgTiO}_{3}$ phase were also observed at reflections of (003), (101). (012), (021), (024) and (300). On the other hand, the diffraction peaks of the remaining starting materials, $\mathrm{TiO}_{2}$ and $\mathrm{MgO}$ became weaker as the sintering increased to $700{ }^{\circ} \mathrm{C}$.

Clearly, at $2 \theta=25^{\circ}, 27^{\circ}, 38^{\circ}, 43^{\circ}$ and $48^{\circ}$, the peaks slowly diminished as the sintering temperature increased. The existence of well-defined peaks, $\mathrm{MgTiO}_{3}$ indicates the crystallization and phase formation occurred in the synthesized compounds. At $800{ }^{\circ} \mathrm{C}$, the sample showed a complete reaction as pure $\mathrm{MgTiO}_{3}$ formed and there was no residual impurities noticed. A progressive improvement in the XRD pattern was observed where the diffraction peaks became stronger, sharper and narrower in intensity at $900{ }^{\circ} \mathrm{C}$. However, there was no major change detected at $1000{ }^{\circ} \mathrm{C}$ to 1400 ${ }^{\circ} \mathrm{C}$ thus further analysis on the FESEM analysis become necessary. Subsequent to 10 hours of sintering process starting from $500{ }^{\circ} \mathrm{C}$ to $1400{ }^{\circ} \mathrm{C}$, magnesium titanate sample were concluded to be improved in crystalline structure as they reached their final sintering state.

Figure 3 shows the surface microstructure photographs of the $\mathrm{MgTiO}_{3}$ samples as a function of sintering temperature from $500{ }^{\circ} \mathrm{C}$ to $1400{ }^{\circ} \mathrm{C}$. At the initial stage of sintering process, there were flat surfaces observed in Figure 3 (a)-(b) which showed they were in amorphous phase with a minimal grain development. Formation of fairly rapid inter-particle neck growth can be seen in Figure 3 (c)-(d). This is in agreement with the diffraction data where at $700{ }^{\circ} \mathrm{C}$, the crystalline structure improved and formed a complete phase at $800{ }^{\circ} \mathrm{C}$. Further increased the temperature, pores were observed and greater conjoint of grains were presented in Figure 3 (e). As tabulated in Table 1, the average grain size increased from $87 \mathrm{~nm}$ to $149 \mathrm{~nm}$ due to the decomposition of raw materials and deficient formation of $\mathrm{MgTiO}_{3}$ caused minimal grain development. The pores were almost eliminated at $1000{ }^{\circ} \mathrm{C}$ to $1200{ }^{\circ} \mathrm{C}$ revealing the samples exhibit an intermediate sintering stage. A relatively uniform surface morphology was observed and higher number of necking process was noticeable in $\mathrm{MgTiO}_{3}$ pellets as perceived in Figure 3 (f)-(h) led to a steeper increase of average grain size from $216 \mathrm{~nm}$ to $526 \mathrm{~nm}$. At the final sintering stages as shown in Figure 3 (i)-(j), the particles grew rapidly into large ones (1537 nm and $2411 \mathrm{~nm}$ ) probably due to a reduction in total interfacial energy. This happened when the sample exhibits its highest densification and largest grain coarsening effect during the sintering process [23].

As shown in Figure 4, the density of the sintered pellets increased concurrently with sintering temperature. The densities observed in $\mathrm{MgTiO}_{3}$ system can be classified into three stages. The samples sintered at $500{ }^{\circ} \mathrm{C}$ to $700{ }^{\circ} \mathrm{C}$ referred as initial stage, showed a sharp increase in density. The increase in density can be explained by correlating their physical properties. From the XRD data, the samples exhibited low crystallization with multiple phases of constituents at $500{ }^{\circ} \mathrm{C}$ to 


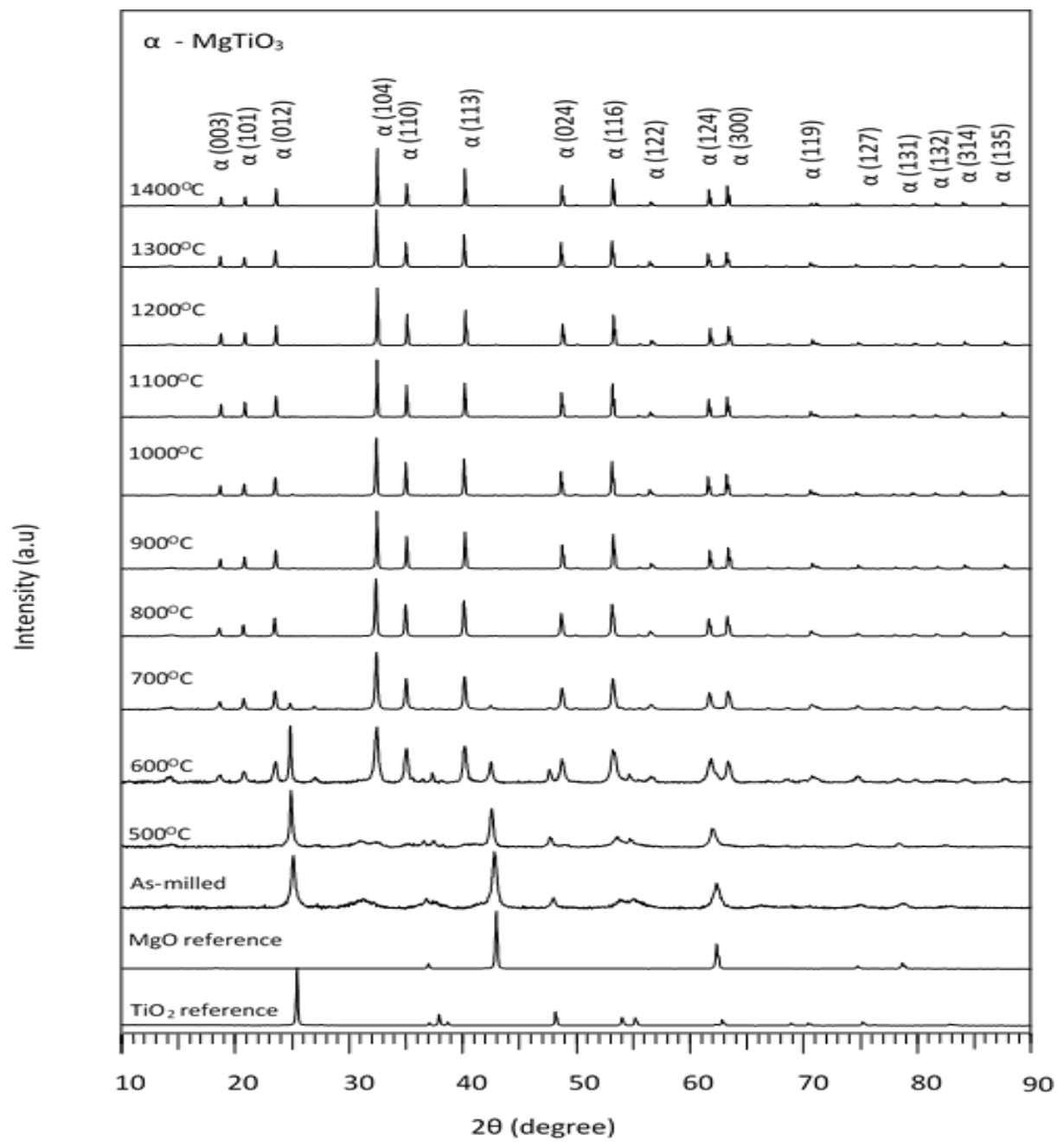

Figure 2: $\mathrm{X}$ - ray diffraction patterns of as-milled powder and magnesium titanate samples sintered from $500{ }^{\circ} \mathrm{C}$ to 1400 ${ }^{\circ} \mathrm{C}$

$700{ }^{\circ} \mathrm{C}$. The highly reactive samples lead to a large surface energy and higher driving force for crystalline growth and finally increased the densification rate. In the intermediate stage $\left(800{ }^{\circ} \mathrm{C}\right.$ to $\left.1200{ }^{\circ} \mathrm{C}\right)$, the samples displayed a steady increase in density, from $3.24 \mathrm{~g} / \mathrm{cm}^{3}$ to $3.48 \mathrm{~g} / \mathrm{cm}^{3}$ shown in Table 1 . By referring to the grain growth of the samples, a higher number of necking process occurred between particles showed a replacement of solid/vapor interfaces by solid/solid interfaces [23]. This caused a reduction of Gibbs energy in the $\mathrm{MgTiO}_{3}$ system thus affecting the increment in densification rate. A significant increase in density is presented at $1300{ }^{\circ} \mathrm{C}$ and $1400{ }^{\circ} \mathrm{C}$ where the density increased to $3.56 \mathrm{~g} / \mathrm{cm}^{3}$ and $3.59 \mathrm{~g} / \mathrm{cm}^{3}$ respectively. Contrary to the initial stage, samples were less reactive since $\mathrm{MgTiO}_{3}$ phase was highly crystalline and large grains with less pores were observed in the surface microstructure image. Thus, the minimization of grain boundary led to an improvement in density $\approx 98.6 \%$ from its theoretical density. 

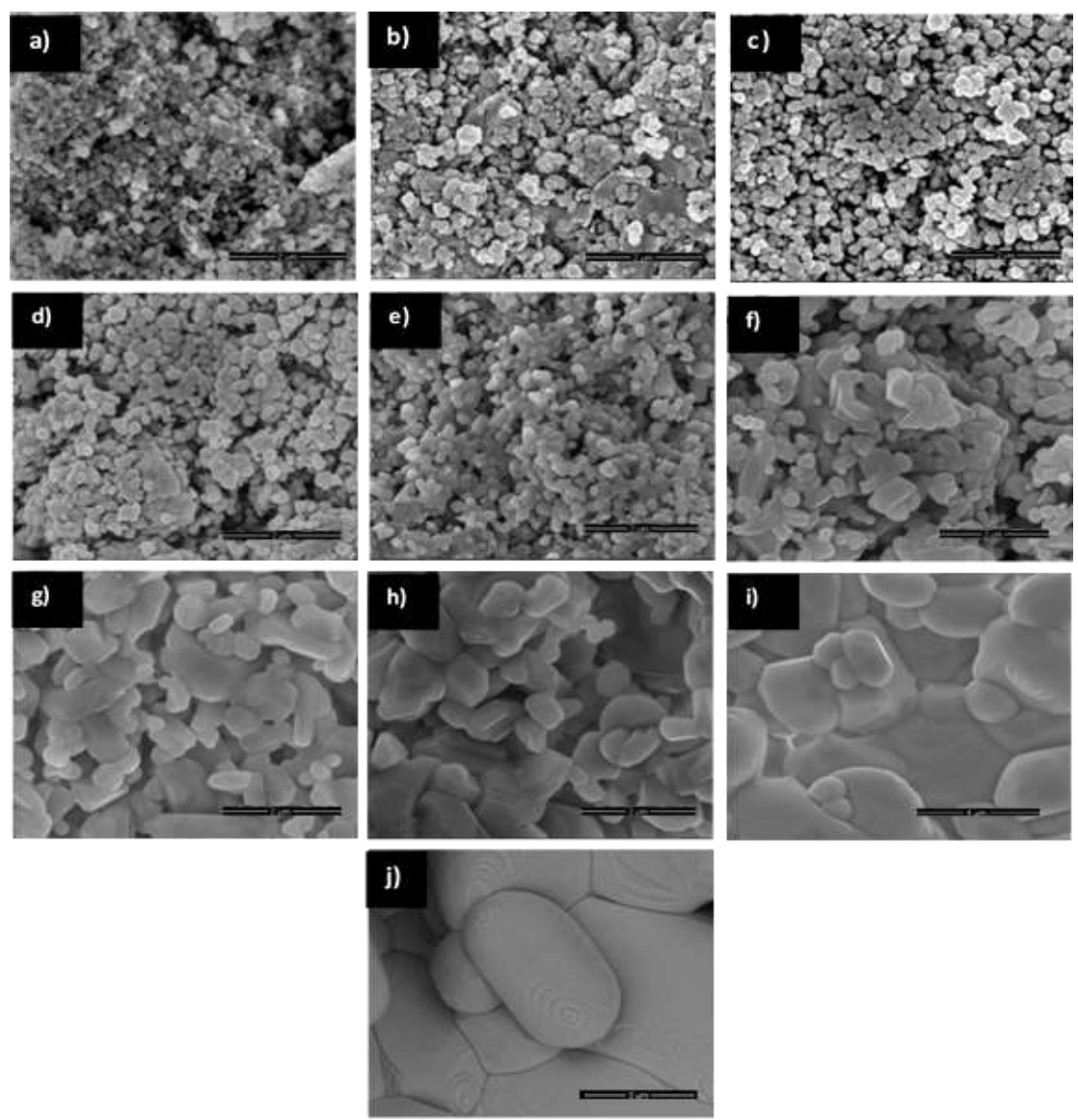

Figure 3: FESEM micrographs of $\mathrm{MgTiO}_{3}$ sintered at (a) $500{ }^{\circ} \mathrm{C}$ (b) $600{ }^{\circ} \mathrm{C}$ (c) $700{ }^{\circ} \mathrm{C}$ (d) $800{ }^{\circ} \mathrm{C}$

(e) $900{ }^{\circ} \mathrm{C}$ (f) $1000{ }^{\circ} \mathrm{C}$ (g) $1100{ }^{\circ} \mathrm{C}$ (h) $1200{ }^{\circ} \mathrm{C}$ (i) $1300{ }^{\circ} \mathrm{C}$ and (j) $1400{ }^{\circ} \mathrm{C}$

Table 1: Experimental density and average grain size of $\mathrm{MgTiO}_{3}$ sample at various sintering temperatures

\begin{tabular}{|c|c|c|c|}
\hline $\begin{array}{c}\text { Sintering } \\
\text { Temperature } \\
\left({ }^{\circ} \mathrm{C}\right) \\
\end{array}$ & $\begin{array}{c}\text { Experimental } \\
\text { density, } \\
\rho_{\exp }\left( \pm 0.01 \mathrm{~g} / \mathrm{cm}^{3}\right)\end{array}$ & $\begin{array}{c}\text { Relative Density, } \\
(\%)\end{array}$ & $\begin{array}{c}\text { Average Grain } \\
\text { Size } \\
( \pm 0.1 \mathrm{~nm})\end{array}$ \\
\hline 500 & 3.08 & 84.0 & 86.8 \\
\hline 600 & 3.12 & 86.6 & 113.1 \\
\hline 700 & 3.24 & 90.6 & 125.9 \\
\hline 800 & 3.28 & 92.5 & 131.1 \\
\hline 900 & 3.33 & 92.4 & 149.1 \\
\hline 1000 & 3.36 & 93.2 & 216.1 \\
\hline 1100 & 3.41 & 93.7 & 332.6 \\
\hline 1200 & 3.48 & 95.9 & 526.3 \\
\hline 1300 & 3.56 & 98.5 & 1537.0 \\
\hline 1400 & 3.59 & 98.6 & 2411.1 \\
\hline
\end{tabular}

\subsection{Complex Permittivity Analysis}

The complex permittivity display valuable information about the conduction process which benefits in understanding the origin of dielectric losses, the electrical and dipolar relaxation time. For this matter, the variation of dielectric constant $\left(\varepsilon_{\mathrm{r}}{ }^{\prime}\right)$ and the loss tangent $(\tan \delta)$ as a function of frequency have been plotted in Figure 4 (a) and (b) respectively. Generally, the value of $\varepsilon_{\mathrm{r}}$ ' for $\mathrm{MgTiO}_{3}$ evolving from $500{ }^{\circ} \mathrm{C}$ up to $1400{ }^{\circ} \mathrm{C}$ measured at room temperature decreased as the frequency increases and attained constant limitation value at higher frequency, at which $\varepsilon_{\infty}$ ' became almost frequency-independent. Strong frequency dependence of $\varepsilon_{\mathrm{s}}$ ' at lower frequency $\left(<10^{4} \mathrm{~Hz}\right)$ can be explained from the Maxwell-Wagner interfacial polarization where there is an accumulation of space charge between the electrodes and the sample contributed from the grain and the grain boundaries under the applied external electric field [26]. However, the reductions of $\varepsilon_{s}^{\prime}$ as the frequency increased shows the decay of polarization in grains because the space charge carrier 
cannot reorient themselves fast enough parallel to the external applied field. As the frequency is further increased, the rapid changes in the direction of electric field caused the lagging in the space charge carrier's directions [27].

It was worth noticing that at frequency beyond $10^{4} \mathrm{~Hz}, \varepsilon_{\infty}$ ' value started to line up in increasing patterns as the samples started to develop (at $500{ }^{\circ} \mathrm{C}$ ) to its final sintering temperature. This gives a better view on the evolution behaviors regarding the polarization-crystallinity relationship. As continuously increased the sintering temperature, larger grains and better crystals growth were promoted, thus reflected higher degree of polarization and produced better $\varepsilon_{\infty}$ ' value. As been discussed in the microstructural section, at lower sintering temperature $\left(<900{ }^{\circ} \mathrm{C}\right)$ the crystal growth was incomplete and there were small grains with higher grain boundaries volume portion. There were higher insulation resistance in the samples and a lot of impurities and defect can diffuse into the grains, making an inhomogeneous grains thus affecting the spontaneous polarization [28].

Below $10^{4} \mathrm{~Hz}, \varepsilon_{\mathrm{s}}$ ' were not in arranged patterns. This is due to the space charged layered induced by several effects such as vacancy defect within the dielectric and large content of impurities and imperfection in the material structures such as pores. The space charge effect became dominant for this type of polarization making the grain size effect less significant in this region.

Figure 4 (b) shows that $\tan \delta$ were dependent on the frequency in the $\mathrm{MgTiO}_{3}$ samples. Initially, the value of tan $\delta$ decreased with increasing frequency and beyond $10^{2} \mathrm{~Hz}, \tan \delta$ increased and attained maximum value before decreased again with value almost zero at higher frequency. At lower frequency, high $\tan \delta$ was probably caused from the decrease orientation of interfacial dipoles or hopping process of charge carriers occur as the electrical current propagate through the sample, which leads to more electrical energy absorption [29].

(a)

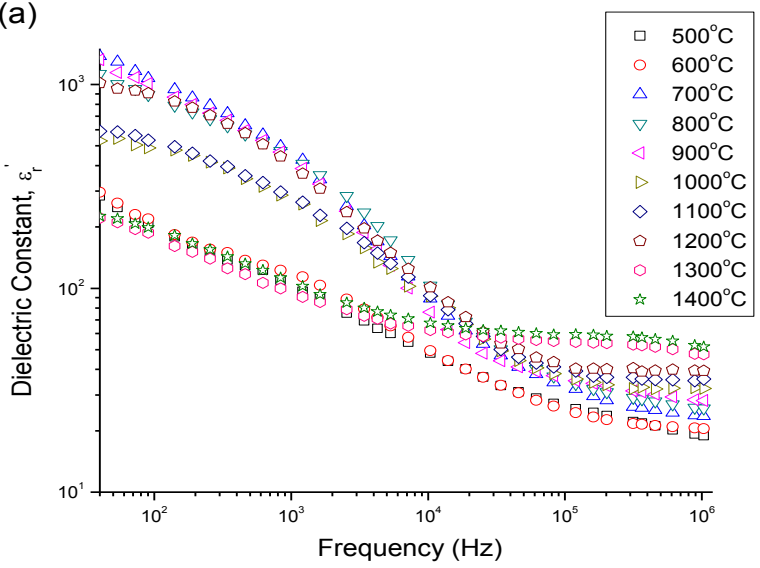

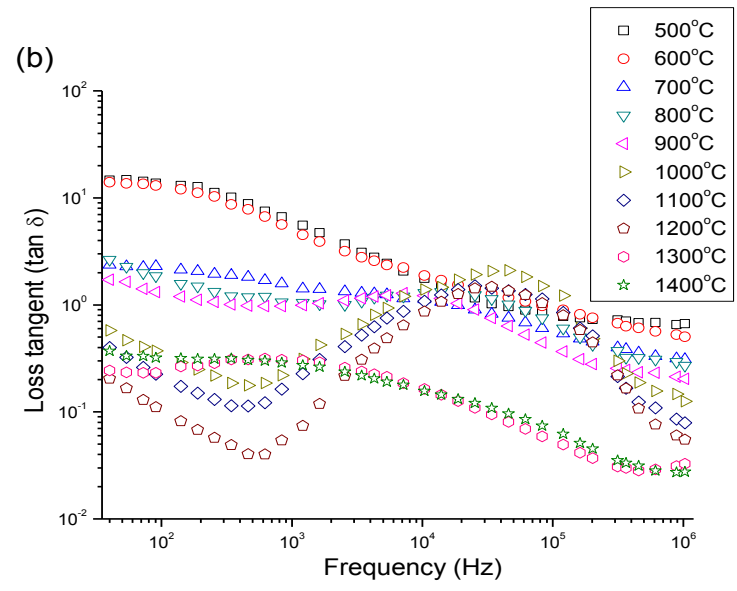

Figure 4: Variation of the dielectric permittivity components (a) $\varepsilon_{\mathrm{r}}^{\prime}$ and (b) tan $\delta$ at room temperature as a function of frequency for $\mathrm{MgTiO}_{3}$ ceramic at different sintering temperatures

\section{CONCLUSION}

$\mathrm{MgTiO}_{3}$ samples have been successfully prepared by mechanical alloying method and sintered from $500{ }^{\circ} \mathrm{C}$ to 1400 ${ }^{\circ} \mathrm{C}$. The diffraction data showed that below $700^{\circ} \mathrm{C}, \mathrm{MgTiO}_{3}$ is in incomplete phase with low crystallization. At $800{ }^{\circ} \mathrm{C}$, $\mathrm{MgTiO}_{3}$ performed a complete phase and there were improvements in the crystalline feature as the sintering temperature is elevated. A particle size distribution of as-milled powder below $100 \mathrm{~nm}$ was observed to increase the rate of grain growth as shown in FESEM. The density and grain size increased with the rise of sintering temperature. The variation of $\varepsilon_{\mathrm{s}}$ ' with frequency showed a strong frequency dependence at lower frequency $\left(<10^{4} \mathrm{~Hz}\right)$ which can be explained from the Maxwell-Wagner interfacial polarization. Loss tangent $(\tan \delta)$ indicated values less than 1 for all samples at $1 \mathrm{MHz}$. The dependency of microstructure growth over the dielectric permittivity were presented and we noticed a significant increasing trends in dielectric constant at $1 \mathrm{MHz}$. Subsequence to the improvement of grain growth and corresponding increase in degree of crystallinity in the material, a higher degree of polarization in grains were perceived. Thus, $\varepsilon_{\infty}$ ' increased proportionally with the microstructure properties at $1 \mathrm{MHz}$.

\section{ACKNOWLEDGEMENT}

This work was financially supported by MyBrain 15 Scholarship. The authors gratefully acknowledge Institute of Advanced Technology (ITMA), and Faculty of Science, Universiti Putra Malaysia for the facilities provided. 


\section{REFERENCES}

[1] B.W. Ricketts, G. Triani, A.D. Hilton, "Dielectric energy storage densities in $\mathrm{Ba}_{1-\mathrm{x}} \mathrm{Sr}_{\mathrm{x}} \mathrm{Ti}_{1-\mathrm{y}} \mathrm{Zr}_{\mathrm{y}} \mathrm{O}_{3}$ ceramics", J Mater Sci - Mater Electron, 11, 513-517, 2000.

[2] A. Belous, O. Ovchar, D. Durylin, M. Valant, M. Macek-Krzmanc, D. Suvorov, "Microwave composite dielectric based on magnesium titanates", J. of the Eur. Ceram. Society, 27, 2963-2966, 2007.

[3] S. Filipovic, N. Obradovic, V.B. Pavlovic, S. Markovic, M. Mitric, M.M. Ristic, "Influence of mechanical activation on microstructure and crystal structure of sintered $\mathrm{MgO}^{-\mathrm{TiO}_{2}}$ system", Sci. of Sintering, 42, 143-151, 2010.

[4] C.L. Pan, C.H. Shen, P.C. Chen, T.C. Tan, "Characterization and dielectric behavior of a new dielectric ceramics $\mathrm{MgTiO}_{3}-\mathrm{Ca}_{0.8} \mathrm{Sr}_{0.2} \mathrm{TiO}_{3}$ at microwave frequencies”, J. of Alloys and Compound, 503, 365-369, 2010.

[5] G. Pfaff, "Peroxide route for synthesis of magnesium titanate powders of various composition", Ceramic International, 20, 111-116, 1994.

[6] N. Obrodovic, S. Filipovic, V.B. Pavlovic, A.Maricic, N. Mitrovic, M.M. Ristic, "Sintering of mechanically activated magnesium-titanate and barium-zinc-titanate ceramics", Sci. of Sintering, 43, 145-151, 2011.

[7] C.L. Huang, C.M. Tsai, A.Yang, A.Hsu, "Compact 5.8-GHz bandpass filter using stepped-impedance dielectric resonators for ISM band wireless communication”, Microwave Opt. Lett., 44, 421, 2005.

[8] J. Bernard, D. Houviet, J.E. Fallah, J.M. Haussonne, " $\mathrm{MgTiO}_{3}$ for $\mathrm{Cu}$ base meral multilayer ceramic capasitors", Jour. of the Eur. Ceram. Soc., Vol 4, 1877-1881, 2004.

[9] K. Sreedhar, N.R. Pavaskar, "Synthesis of $\mathrm{MgTiO}_{3}$ and $\mathrm{Mg} 4 \mathrm{Nb} 2 \mathrm{O}_{9}$ using stoichiometrically excess $\mathrm{MgO}$ ", $\mathrm{Mater}$. Lett., 53, 452-455, 2003.

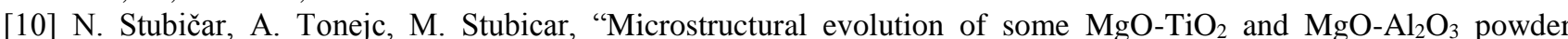
mixtures during high-energy ball milling and post-annealing studied by X-ray diffraction”, J. of Alloys Comp., 370, 296-301, 2004.

[11] I.R. Abothu, A.V.P. Rao, S. Komarneni, "Nanocomposite and monophasic synthesis routes to magnesium titanate", Mater. Lett., 38, 186-189, 1999.

[12] V.M. Ferreira, J.L. Baptista, "Preparation and microwave dielectric properties of pure and doped magnesium titanate ceramics", Mater. Res. Bull., 29, 1017-1023, 1994.

[13] Y. Miao, Q. Zhang, H. Yang, H. Wang, "Low-temperature synthesis of nano-crystalline magnesium titanate materials by the sol gel method", Mater. Sci. and Eng. B, 128, 103-106, 2006.

[14] W.W. Cho, K.I. Kakimoto, H. Ohsato, "Microwave dielectric properties and low-temperature sintering of $\mathrm{MgTiO}_{3}-$ $\mathrm{SrTiO}_{3}$ ceramics with $\mathrm{B}_{2} \mathrm{O}_{3}$ or $\mathrm{CuO}$ ”, Mater. Sci. Eng. B, 121, 48-53, 2005.

[15] J. Liao, M. Senna, "Crystallization of titania and magnesium titanate from mechanically activated $\mathrm{Mg}(\mathrm{OH})_{2}$ and $\mathrm{TiO}_{2}$ gel mixture", Mater. Res. Bull., 30, 385-392, 1995.

[16] B.D. Lee, H.R. Lee, K.H. Yoon, Y.S Cho, "Microwave dielectric properties of magnesium calcium titanate thin films", Ceramic Inter., 31, 143-146, 2005.

[17] D. Li, L. Wang, D. Xue, "Stearic acid gel derived $\mathrm{MgTiO}_{3}$ nanoparticles: A low temperature intermediate phase of $\mathrm{Mg}_{2} \mathrm{TiO}_{4}$ ", Jour. of Alloys and Comp., 492, 564-569, 2010.

[18] C.C., Koch, O.B., Cavin, C.G. McCamey, J.O. Scarborough, "Preparation of amorphous $\mathrm{Ni}_{60} \mathrm{Nb}_{40}$ by mechanical alloying", Appl. Phys. Lett., 43, 1017, 1983.

[19] C. Suryanarayana, Mechanical Alloying and Milling, New York: Marcel Dekker, 2004.

[20] M.A. Sanoj, C.P. Reshmi, K.P. Sreena, M.R. Varma, "Sinterability and microwave dielectric properties of nano structured $0.95 \mathrm{MgTiO}_{3}-0.05 \mathrm{CaTiO}_{3}$ synthesised by top down and bottom up approaches", J. of Alloys and Comp., 509, 3089-3095, 2011.

[21] V. Petrovic, "Sintering kinetics of $\mathrm{MgO}-\mathrm{TiO}_{2}$ systems", Sci. of sintering ceramics, 38, 287-292, 2006.

[22] J. Widegren, L. Bergstrom, "Electrostatic stabilization of ultrafine titania in ethanol”, J. Am Ceram. Soc., 85, 523$528,2002$.

[23] S. Joong, L. Kang, Sintering Densification, Grain Growth and Microstructure, Elsevier/Butterworth-Heinemann, London, 2005.

[24] I. Ismail, M. Hashim, "Sintering temperature dependence of evolving morphologies and magnetic properties of $\mathrm{Ni}_{0.5} \mathrm{Zn}_{0.5} \mathrm{Fe}_{2} \mathrm{O}_{4}$ synthesized via mechanical alloying", J. Supercond Nov. Magnetic, 25, 1551-1561, 2012.

[25] R. L. Coble, "Sintering crystalline solids. II. Experimental test of diffusion models in powder compact", Jour. Applied Phy., 32, 793-799, 1961.

[26] A.A. Saif, P. Poopalan, "Correlation between the chemical composition and the conduction mechanism barium strontium titanate thin films", J. of Alloys and Comp., 509, 7210-7215, 2011.

[27] M. Chanda, Science of engineering materials, Vol. 3, the Macmillan Company of India Ltd. 1980.

[28] C. Mao, S. Yan, S. Cao, C. Yao, F. Cao, G. Wang, X. Dong, X. Hu, C. Yang, "Effect of grain size on phase transition, dielectric and pyroelectric properties of BST ceramics", J. Of Eur. Ceramic Soc., 34,) 2933-2939, 2014.

[29] R.V. Mangalaraja, P. Manohar, F.D. Gnanam, M.Awano, "Electrical and magnetic properties of $\mathrm{Ni}_{0.8} \mathrm{Zn}_{0.2} \mathrm{Fe}_{2} \mathrm{O}_{4}$ /silica composite prepared by sol-gel method”, J. Mater. Sci., 39, 2037, 2004. 